\title{
InGaN Micro-LED-Pillar as the Building Block for High Brightness Emitters
}

\author{
Chao Shen ${ }^{1}$, Tien Khee $\mathrm{Ng}^{1}$, Yang Yang ${ }^{2}$, Dongkyu Cha ${ }^{2}$ and Boon S. Ooi ${ }^{1}$ * \\ ${ }^{1}$ Photonics Laboratory, King Abdullah University of Science and Technology, Thuwal, 21534, Saudi Arabia \\ ${ }^{2}$ Advanced Nanofabrication Imaging and Characterization Laboratory (ANIC), King Abdullah University of Science and \\ Technology, Thuwal, 21534, Saudi Arabia \\ *Corresponding E-mail: boon.ooi@kaust.edu.sa
}

\section{INTRODUCTION}

The origin of efficiency droop in the InGaN/GaN MQW LEDs has been widely discussed and studied $[1,2]$. InGaN-QW-based micro-LED structure ( $\mu$ LED-pillar henceforth), used in visible light communication [3] and micro-display [4], were demonstrated to hold unique electrical characteristics suitable for high power LED lighting. It was reported that $\mu$ LED-pillar with diameter $(D)<100 \mu m$ can sustain high injection current density, and showed an improved efficiency at high drive current [5]. A reduced efficiency droop effect can hence be expected in $\mu$ LED-pillars. In this paper, we investigate the effect of strain relaxation in the etched micro-pillar on the efficiency droop of LED. A significant reduction of efficiency droop has been observed from the $\mu$ LEDpillars.

\section{EXPERIMENTS}

The LED structure was grown on c-plane sapphire substrate with 12 -stack $3 \mathrm{~nm}$-InGaN-well / 10nm-GaNbarrier multiple quantum wells (MQWs) as the active layer (see Fig. 1). Both large-area LEDs $(D=200 \sim 500$ $\mu \mathrm{m})$ and $\mu$ LED-pillars $(D=20 \sim 80 \mu \mathrm{m})$ were fabricated using UV contact-lithography and $\mathrm{Cl}_{2}$ based inductivelycoupled plasma (ICP) etching. Photoluminescence (PL) was measured using a $325 \mathrm{~nm} \mathrm{HeCd}$ laser at room temperature.

\section{RESULTS AND DISCUSSION}

Figure 2(a) shows the injection current density $v s$. the DC bias characteristics for the $\mu$ LED-pillars compared with a large-area LED $(D=200 \mu \mathrm{m})$. As expected, a higher current density of $492 \mathrm{~A} / \mathrm{cm}^{2}$ was measured from the $20 \mu m \mu$ LED-pillar, compared with that of $200 \mu \mathrm{m} \operatorname{LED}\left(20 \mathrm{~A} / \mathrm{cm}^{2}\right)$ at bias voltage of $10 \mathrm{~V}$. The external quantum efficiencies (EQEs) were calculated by measuring the light output powers under different injection current density. The strong sizedependence in efficiency droop is evident in Fig. 2(b), where the peak EQE $\left(\eta_{\text {peak }}\right)$ occurs at a higher injection current density for smaller LED. With increasing current, the EQE steadily drops beyond $\eta_{\text {peak }}$. (a)

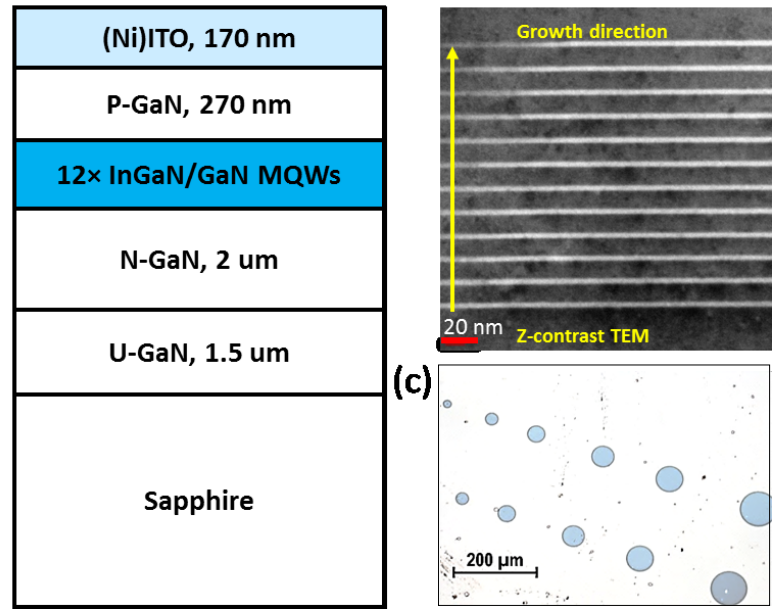

Fig. 1. (a) LED epitaxy with sputtered (Ni)-ITO p-contact. (b) Zcontrast TEM micrograph of 12-stack InGaN/GaN MQWs. (c) Optical microscope image of two rows of $\mu$ LED-pillars.
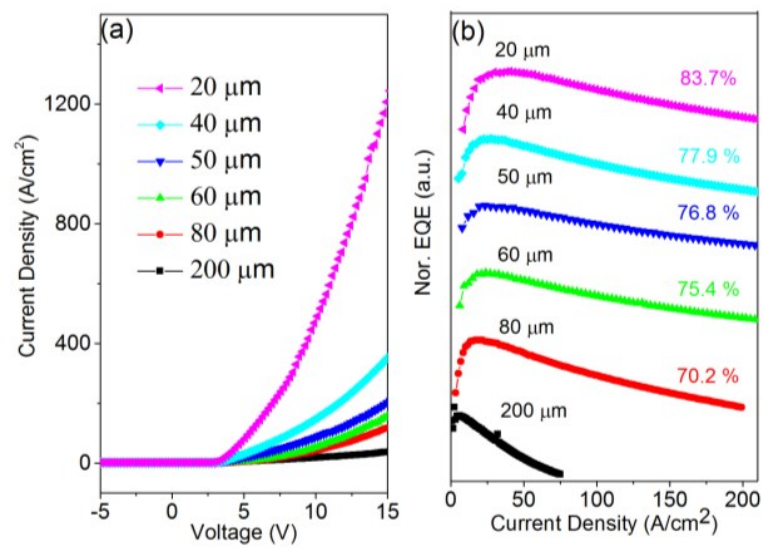

Fig. 2. (a) I-V measurements of $\mu$ LED-pillars and a large-area LED, (b) Normalized external quantum efficiency (EQE) vs. injection current density in various $\mu$ LED-pillars compared with a large-area LED. The $\eta_{200} / \eta_{\text {peak }}$ of each $\mu$ LED-pillar is indicated.

To realize a high brightness LED, the ability to sustain high quantum efficiency under large injection current density is required. Comparing the ratios of sustained EQE at $200 \mathrm{~A} / \mathrm{cm}^{2}\left(\eta_{200} / \eta_{\text {peak }}\right)$, we observed a 
reduction of efficiency droop in small $\mu$ LED-pillars. Over $80 \% \eta_{\text {peak }}$ was sustained in the $20 \mu \mathrm{m} \mu \mathrm{LED}$-pillar, compared to only $70 \%$ in that of $80 \mu \mathrm{m} \mu \mathrm{LED}$-pillar. We further explore the reason for such observation in the following.

Enhanced current spreading resulting in the improvements of electrical properties [6] and EQE [7] in LEDs, may contribute to a reduction in efficiency droop. According to the current density distribution theory [8], the lateral current spreading length, $L_{s}$, is:

$$
L_{s}=\sqrt{\left(\rho_{c}+\rho_{p} t_{p}\right) t_{n} / \rho_{n}}
$$

which is defined as the length at the point where the lateral current density is reduced to $1 / \mathrm{e}$ of the current density at the pillar edge. For our case, $L_{s}$ is calculated to be $55 \mu \mathrm{m}$, and the fraction of current density at the center of the pillar to that at the edge are $0.83,0.63$ and 0.48 for devices with $D=20 \mu \mathrm{m}, 50 \mu \mathrm{m}$ and $80 \mu \mathrm{m}$, respectively. Thus a uniform current spreading (larger fraction) in smaller $\mu$ LED-pillar results in the improved electrical property. Furthermore, with a reduced size, the current crowding effect at the active region is less significant, leading to reduced Auger recombination probability, and local heating [9].

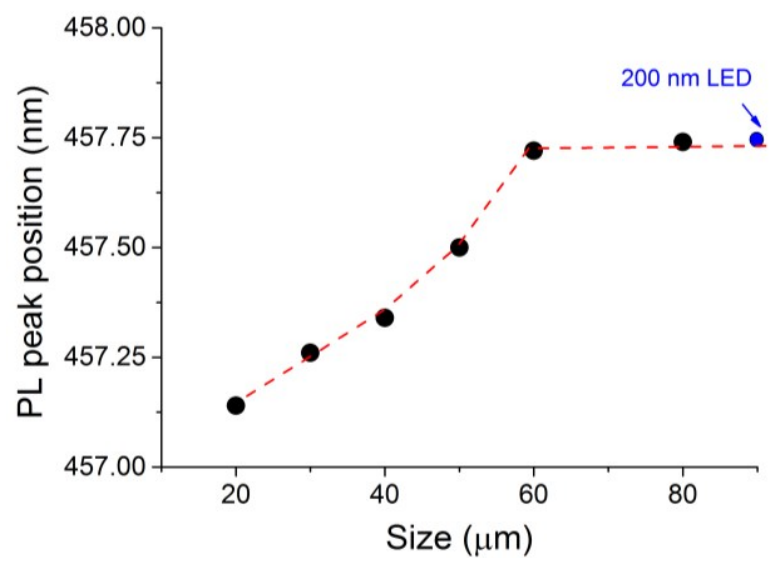

Fig. 3. PL peak positions measured from the center of each $\mu$ LEDpillar as a function of the pillar size.

Our subsequent investigation reveal that strain relief plays an important part in sustaining EQE at high injection current, notably in small $\mu$ LED-pillars. To evaluate the potential strain relaxation, PL spectra were collected and the peak positions were plotted in Fig. 3. No observable PL emission peak shift was found for $\mu$ LED-pillars with $D>50 \mu \mathrm{m}$, but a blue shift $(\sim 4$ meV) was identified in $\mu$ LED-pillar with $D=20 \mu \mathrm{m}$. The peak shift trend evidences that strain relief is not negligible in $\mu$ LED-pillars with $D<50 \mu \mathrm{m}$. Our investigation suggests that $\mu$ LED-pillars with $D<20$ $\mu m$, benefitting from both the uniform current spreading and the strain relief, will have further improvement in electrical and quantum efficiency characteristics.

\section{CONCLUSIONS}

In summary, we confirmed the improved electrical and optical characteristics, with reduced efficiency droop in InGaN $\mu$ LED-pillars when these devices were scaled down in size. We demonstrated that strain relief contributed to further improvement in $E Q E$ characteristics in small InGaN $\mu$ LED-pillars $(D<50$ $\mu m$ ), apart from the current spreading effect. The $\mu$ LEDpillar can be deployed as the building block for large effective-area, high brightness emitter.

\section{REFERENCES}

[1] J. Iveland et al., Direct Measurement of Auger Electrons Emitted from a Semiconductor Light-Emitting Diode under Electrical Injection: Identification of the Dominant Mechanism for Efficiency Droop. Phys Rev Lett 110, 177406 (2013).

[2] Y. L. Li et al., Investigation of Efficiency Droop Behaviors of InGaN/GaN Multiple-Quantum-Well LEDs With Various Well Thicknesses. IEEE J Sel Top Quant 15, 1128 (2009).

[3] L. Zhu et al., in Photonics Global Conference (PGC), 2010, pp. $1-3$.

[4] Z. Shuailong et al., in Photonics Conference (IPC), 2012 IEEE, pp. $435-436$.

[5] P. F. Tian et al., Size-dependent efficiency and efficiency droop of blue InGaN micro-light emitting diodes. Appl Phys Lett 101, 231110 (2012)

[6] H. Kim et al., Measurements of current spreading length and design of GaN-based light emitting diodes. Appl Phys Lett 90, 063510 (2007).

[7] Y. B. Tao et al., Size effect on efficiency droop of blue light emitting diode. Phys Stat Soli C Vol 9, No 3-4 9, 616 (2012).

[8] X. Guo, E. F. Schubert, Current crowding and optical saturation effects in $\mathrm{GaInN} / \mathrm{GaN}$ light-emitting diodes grown on insulating substrates. Appl Phys Lett 78, 3337 (2001).

[9] V. K. Malyutenko, S. S. Bolgov, A. D. Podoltsev, Current crowding effect on the ideality factor and efficiency droop in blue lateral $\mathrm{InGaN} / \mathrm{GaN}$ light emitting diodes. Appl Phys Lett 97, $251110(2010)$ 